\title{
PROPRIEDADES QUÍMICAS DE UM LATOSSOLO VERMELHO IMPLANTADO COM PASTOS DE CAPIM-MOMBACCA SOB EFEITO RESIDUAL DO NITROGÊNIO
}

\author{
PROPIEDADES QUÍMICAS DE UN LATOSOL ROJO IMPLANTADO CON CAPIM- \\ MOMBAÇA PASTAS BAJO EFECTO RESIDUAL DE NITRÓGENO
}

\section{CHEMICAL PROPERTIES OF A RED LATOSOL IMPLANTED WITH CAPIM- MOMBAÇA PASTES UNDER RESIDUAL EFFECT OF NITROGEN}

Antonio Leandro Chaves Gurgel ${ }^{1}$; Gelson dos Santos Difante ${ }^{2}$; Alexandre Romeiro de Araújo $^{3}$; Manuel Cláudio Motta Macedo ${ }^{4}$; Denise Baptaglin Montagner ${ }^{5}$

DOI: https://doi.org/10.31692/978-65-991061-4-9.127-132

\section{INTRODUÇÃO}

O principal fator responsável por tornar a bovinocultura de corte brasileira competitiva em nível mundial é a produção quase que exclusivamente em pasto. A adubação nitrogenada, juntamente com a adubação de manutenção com os demais nutrientes essenciais às plantas são fundamentais para garantir a produtividade agropecuária (LIU et al., 2010; ARLAUSKIENE் et al., 2019). O uso de nitrogênio aumenta a produtividade das pastagens e permite intensificar a utilização por meio de maiores taxas de lotação (MOREIRA et al., 2011).

Essa maior produção de forragem observada com a adoção de maiores doses de nitrogênio (BERNADI et al., 2018) promove maior extração e incorporação de nutrientes no tecido vegetal (MAGALHÃES et al., 2002). Soma-se a isso a condição dos solos de Cerrado, que são considerados de baixa fertilidade natural, acidez elevada e baixo teor de matéria orgânica (BRAZ et al., 2004), o que torna essencial os cuidados com a fertilidade do solo. Por outro lado, há escassez de informações na literatura que avaliem o efeito residual de doses de nitrogênio nos atributos químicos do solo.

Diante do exposto, o objetivo foi avaliar o efeito residual de doses de nitrogênio nas características químicas de um Latossolo vermelho implantado com pastos de capimmombaça.

\section{FUNDAMENTAÇÃO TEÓRICA}

O nitrogênio é o elemento mais abundante na atmosfera terrestre (70\%), e exigido em maior quantidade pela maioria das plantas, desempenha inúmeras funções e constitui

\footnotetext{
${ }^{1}$ Doutorando em Ciência Animal, Universidade Federal de Mato Grosso do Sul, antonioleandro09@gmail.com

2 Professor, Doutor, Universidade Federal de Mato Grosso do Sul, gdifante@ hotmail.com

${ }^{3}$ Pesquisador, Doutor, EMPRAPA Gado de Corte, alexandre.araujo@embrapa.br

${ }^{4}$ Pesquisador, Doutor, EMPRAPA Gado de Corte, manuel.macedo@embrapa.br

${ }^{5}$ Pesquisadora, Doutora, EMPRAPA Gado de Corte, Denise.montagner@embrapa.br
} 
molécula de importância para a vida na terra. Especificamente na planta, é um dos mais importantes constituintes de aminoácidos, proteínas, ácidos nucléicos e enzimas; promove o crescimento e dá a pigmentação verde das plantas (TAIZ e ZEIGUER, 2006).

Estima-se que apenas $47 \%$ do nitrogênio adicionado globalmente aos solos é convertido e colhido em forma de produtos (LASSALETTA et al., 2014). Mais de 50\% do N é perdido no meio ambiente (FOYER et al., 2016), o que gera desperdiço de recursos financeiros, ameaças os corpos de águas, a biodiversidade e aumenta emissões de gases poluentes (GODINOT et al. 2014). Esses fatos tornam imprescindível que nos sistemas pecuários atuais sejam adotadas medidas potenciais que promovam a máxima eficiência de uso desse nutriente. Tendo em vista a sua reconhecida essencialidade para produção de alimentos (ŚWITEK et al., 2019).

A fertilidade do solo desempenha um papel importante no desenvolvimento e produtividade das plantas. Magalhães et al. (2002) avaliaram as relações entre produção de forragem e exportação de nutrientes, em solos sob cerrado durante seis anos de utilização com Brachiaria brizantha, e observaram que a produção de forragem altera a quantidade de nutrientes exportada. Malavolta (1980) demonstrou valores de produção forragem que

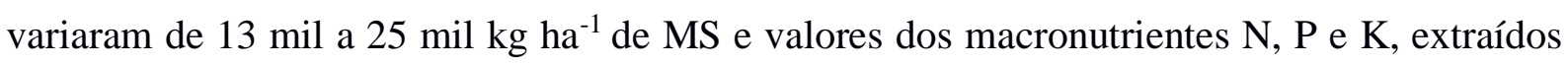
pela parte aérea de, respectivamente, 200 a $300 \mathrm{~kg} \mathrm{ha}^{-1}, 30$ a $70 \mathrm{~kg} \mathrm{ha}^{-1}$ e 200 a $500 \mathrm{~kg} \mathrm{ha}^{-1}$. Portanto, quanto maior a intensificação do sistema, maiores os cuidados com a fertilidade do solo.

\section{METODOLOGIA}

O experimento foi realizado na Embrapa Gado de Corte, em Campo Grande, MS $\left(20^{\circ} 27^{\prime} \mathrm{S}\right.$ e $54^{\circ} 37^{\prime} \mathrm{W}$, a $530 \mathrm{~m}$ de altitude). O período experimental foi de 20/11/2017 a 22/05/2018. O clima da região, segundo a classificação de Köppen, é do tipo AW, tropical chuvoso de savana. A área experimental possui 13,5 ha, dividida em três blocos, cada bloco foi subdividido em três módulos de 1,5 ha, e estes, em seis piquetes de 0,25 ha cada. O solo da área experimental é classificado como Latossolo Vermelho (Embrapa, 2013), com teores de argila entre 30 e $35 \%$.

Os pastos receberam três doses anuais de nitrogênio na forma de ureia (100, 200 e 300 $\mathrm{kg} \mathrm{ha}^{-1} \mathrm{de} \mathrm{N}$ ) por três anos consecutivos 2014/2015, 2015/2016 e 2016/2017, além de adubação de cobertura com $80 \mathrm{~kg} \mathrm{ha}^{-1}$ de $\mathrm{P}_{2} \mathrm{O}_{5}$ e $80 \mathrm{~kg} \mathrm{ha}^{-1}$ de $\mathrm{K}_{2} \mathrm{O}$. A partir das águas de 2017 não foi utilizada adubação de manutenção, nem nitrogenada, caracterizando a avaliação do efeito residual das doses de nitrogênio. Para desfolhação foram utilizados bovinos machos 
inteiros da raça Nelore. Os pastos foram manejados pelo método de pastejo com lotação intermitente, com taxa de lotação variável de modo a garantir uma altura pós pastejo de 40 a $50 \mathrm{~cm}$ (EUCLIDES et al., 2017). Nos cinco primeiros ciclos de pastejo (verão), foi preconizado cinco dias de ocupação e 25 dias de descanso, no sexto ciclo (outono), os pastos foram manejados com sete dias de ocupação e 35 dias de descanso.

As diferentes doses residuais de nitrogênio proporcionaram taxas de acúmulo de forragem distintas entre os tratamentos que, por sua vez, definiram diferentes taxas de lotação no decorrer do período experimental (Tabela 1).

Tabela 1. Indicadores médios de produção animal e de forragem, em pastos de capim-mombaça sob efeito residual de nitrogênio.

\begin{tabular}{cccc}
\hline Variáveis & \multicolumn{3}{c}{ Doses de $\mathrm{N}\left(\mathrm{kg} \mathrm{ha}^{-1} \mathrm{de} \mathrm{N}\right)$} \\
\cline { 2 - 4 } & 100 & 200 & 300 \\
\hline Taxa de acúmulo de forragem $\left(\mathrm{kg} \mathrm{ha}^{-1}\right.$ por dia) & 26,7 & 36,3 & 43,4 \\
Massa de forragem no pré-pastejo $\left(\mathrm{kg} \mathrm{ha}^{-1} \mathrm{MS}\right)$ & 3371,8 & 3637,6 & 3853,9 \\
Taxa de lotação $(\mathrm{UA} \mathrm{ha})^{-1}$ & 2,7 & 3,1 & 4,0 \\
\hline
\end{tabular}

*UA= Unidade anima de $450 \mathrm{~kg}$. Fonte: Própria (2019).

As amostras de solo para análise química foram coletadas em maio de 2018, em três profundidades ( 0 a 10, a 20 e 20 a $40 \mathrm{~cm}$ ). Para a coleta foram escavadas trincheiras com um metro de profundidade em dois piquetes de cada bloco experimental. As amostra de solo foram armazenadas em saco plástico, identificadas e encaminhadas ao Laboratório de Solos da Empresa Brasileira de Pesquisa Agropecuária (EMBRAPA - Gado de Corte). Os atributos químicos avaliados foram: $\mathrm{pH} \mathrm{CaCl}_{2}$ (proporção 1: 2,5), acidez total $\mathrm{pH} 7,0(\mathrm{H}+\mathrm{Al})$, teor de fósforo, potássio trocável, cálcio trocável, magnésio trocável, saturação por bases, capacidade de troca catiônica $(\mathrm{CTC}$ em $\mathrm{pH}=7,0)$, e teor de matéria orgânica, conforme a metodologia de Embrapa, (2017).

O delineamento adotado foi o de casualização em blocos com arranjo em parcelas subdivididas, sendo o efeito residual das doses de nitrogênio alocado na parcela e as profundidades na subparcela. Os dados foram submetidos à análise de variância, quando significativos pelo teste $\mathrm{F}$, o efeito residual do nitrogênio, das profundidades e suas interações, foram analisados pelo teste de Tukey, a 5\% de significância.

\section{RESULTADOS E DISCUSSÃO}

A interação entre dose residual de nitrogênio $\times$ profundidade do solo não foi significativa para as variáveis estudadas (Tabela 2). Não houve efeito das doses residuais de 
nitrogênio para o $\mathrm{pH}$ do solo, cálcio trocável, potássio trocável, acidez potencial, capacidade de troca catiônica, saturação por bases e fósforo. No entanto, as doses residuais de nitrogênio influenciaram os teores de magnésio trocável, com as maiores concentrações no solo sob efeito das doses residuais de 100 e $200 \mathrm{~kg} \mathrm{ha}^{-1}$ de $\mathrm{N}$, e de matéria orgânica, com os maiores valores no solo sob efeito das doses residuais de 200 e $300 \mathrm{~kg} \mathrm{ha}^{-1}$ de $\mathrm{N}$ (Tabela 2).

As pequenas alterações nos atributos químicos do solo, ocorreram, provavelmente, devido à similaridade do manejo da adubação de manutenção adotado nos anos que antecederam a coleta de dados. Por outro lado, os pastos sob efeito da dose residual de 300 $\mathrm{kg} \mathrm{ha}^{-1}$ de $\mathrm{N}$ acumularam mais forragem (Tabela 1) o que levou a maior extração de magnésio do solo, pois, as gramíneas forrageiras são mais eficientes na absorção e acumulação de magnésio nos tecidos (BRAZ et al., 2004; ANDREOLE e PRADO, 2012; ENSINAS et al., 2016).

Tabela 1. Características químicas de um Latossolo Vermelho implantado com pastos de capim-mombaça sob efeito residual do nitrogênio em diferentes profundidades.

\begin{tabular}{|c|c|c|c|c|c|c|c|c|c|c|}
\hline \multirow{2}{*}{ Propriedades } & \multicolumn{3}{|c|}{ Doses de $\mathrm{N}\left(\mathrm{Kg} \mathrm{ha}^{-1}\right)$} & \multicolumn{3}{|c|}{ Profundidades $(\mathrm{cm})$} & \multirow{2}{*}{ EPM } & \multicolumn{3}{|c|}{ Valor P } \\
\hline & 100 & 200 & 30 & 0 a 10 & 0 a 20 & 20 a 40 & & $\mathrm{~N}$ & $\mathrm{P}$ & $\mathrm{N} \times \mathrm{P}$ \\
\hline $\mathrm{pH}\left(\mathrm{CaCl}_{2}\right)$ & 5,3 & 5,3 & & 5,2 & 5,3 & 5,3 & 0,024 & 0,182 & 0,074 & 0,855 \\
\hline Cálcio $\left(\mathrm{cmol}_{\mathrm{c}} \mathrm{dm}^{-3}\right)$ & 2,3 & 2,1 & 2,0 & $2,6^{\mathrm{a}}$ & $2,5^{\mathrm{a}}$ & $1,3^{b}$ & 0,097 & 0,223 & 0,001 & 0,393 \\
\hline Magnésio $\left(\mathrm{cmol}_{\mathrm{c}} \mathrm{dm}^{-3}\right)$ & $1,2^{\mathrm{a}}$ & $1,2^{\mathrm{a}}$ & 1 & $1,3^{\mathrm{a}}$ & $1,2^{\mathrm{a}}$ & $0,9^{\mathrm{b}}$ & 0,017 & 0,022 & 0,001 & 0,931 \\
\hline Potássio $\left(\mathrm{cmol}_{\mathrm{c}} \mathrm{dm}^{-3}\right)$ & 0,2 & 0,2 & 0,2 & $0,3^{\mathrm{a}}$ & $0,3^{\mathrm{a}}$ & 0,1 & 0,012 & 0,337 & 0,001 & 0,732 \\
\hline $\mathrm{H}+\mathrm{AL}\left(\mathrm{cmol}_{\mathrm{c}} \mathrm{dm}\right.$ & 3,4 & 3 & 3,8 & $4,1^{\mathrm{a}}$ & $3.4^{\mathrm{b}}$ & $3,0^{\mathrm{b}}$ & 0,141 & 0,183 & 0,001 & 0,169 \\
\hline $\mathrm{CTC}\left(\mathrm{cmol}_{\mathrm{c}} \mathrm{dm}^{-3}\right)$ & 6,9 & 7,0 & 7,1 & $8,4^{\mathrm{a}}$ & $7,4^{\mathrm{b}}$ & $5,3^{\mathrm{c}}$ & 0,068 & 0,415 & 0,001 & 0,943 \\
\hline $\mathrm{V}(\%)$ & 51,6 & 49,8 & 46,6 & $50,9^{\mathrm{a}}$ & $54,0^{\mathrm{a}}$ & $43,0^{\mathrm{b}}$ & 1,372 & 0,135 & 0,002 & 0,629 \\
\hline Matéria orgânica (\%) & $3,2 b$ & $3,5^{\mathrm{a}}$ & $3,6^{\mathrm{a}}$ & $4,1^{\mathrm{a}}$ & $3,7^{\mathrm{b}}$ & $2,5^{\mathrm{c}}$ & 0,411 & 0,005 & 0,001 & 0,913 \\
\hline Fósforo $\left(\mathrm{mg} \mathrm{dm}^{-3}\right)$ & 3,2 & 3,9 & 5,0 & $7,4^{\mathrm{a}}$ & $3,7^{\mathrm{b}}$ & $0,9^{c}$ & 0,738 & 0,323 & 0,001 & 0,101 \\
\hline
\end{tabular}

Capacidade de troca catiônica (CTC), saturação por bases (V). Valor de probabilidade (valor P) para as doses de nitrogênio $(\mathrm{N})$ e profundidades $(\mathrm{P})$. EPM: erro padrão da média. Médias seguidas de letras distintas na linha diferem entre si $(\mathrm{P}>0,05)$ pelo teste de Tukey. Fonte: Própria (2019).

O efeito das profundidades não foi significativo para o $\mathrm{pH}$ do solo. A acidez potencial $(\mathrm{H}+\mathrm{Al})$ apresentou maiores valores nas profundidades de 0 a 10 e 10 a $20 \mathrm{~cm}$. Mesma tendência observada para os teores de potássio trocável, cálcio trocável, magnésio trocável, e saturação por bases. A capacidade de troca catiônica, os teores de fósforo e de matéria orgânica diminuíram com o aumento da profundidade (Tabela 2). A redução nos atributos químicos do solo com o aumento em profundidade, pode ser explicada pela particularidade dos sistemas de produção em pastos tropicais, onde não ocorre a incorporação de nutrientes em camadas mais profundas por meio de adubações de cobertura. Essa prática ocorre, geralmente, apenas na implantação do pasto (MAGALHÂES et al., 2002). 


\section{CONCLUSÕES}

As doses residuais de nitrogênio tiveram pouca interferência nos atributos químicos do Latossolo Vermelho de textura média cultivado com capim-mombaça. Ocorreram modificações apenas para os teores de magnésio trocável e matéria orgânica no solo.

\section{REFERÊNCIAS}

ANDRIOLE, I.; PRADO, R. M. Plantas de cobertura em pré-safra e adubação nitrogenada na fertilidade do solo em diferentes camadas, cultivado com milho em sistema de plantio direto e convencional. Semina Ciências Agrarias. v. 33, n. 3, p. 963-978. 2012.

ARLAUSKIENĖA, A.; CESEVIČIENĖB, J.; VELYKISA, A. Improving mineral nitrogen control by combining catch crops, fertilisation, and straw management in a clay loam soil. Acta Agriculturae Scandinavica, Section B - Soil \& Plant Science. v. 69, n. 1, p. 422-431. 2019.

BERNARDI, A.; SILVA, A. W. L.; BARETTA, D. Estudo metanalítico da resposta de gramíneas perenes de verão à adubação nitrogenada. Arquivos Brasileiro de Medicina Veterinária e Zootecnia. v. 70, n. 2, p .545-553, 2018.

BRAZ, A. J. B. P.; SILVEIRA, P. M.; KLIEMANN, H. J.; ZIMMERMANN, F. J. P.; Acumulação de nutrientes em folhas de milheto e dos capins braquiária e mombaça. Pesquisa Agropecuária Tropical. v. 34, n. 2, p. 83-87. 2004.

EMBRAPA - EMPRESA BRASILEIRA DE PESQUISA AGROPECUÁRIA. Centro Nacional de Pesquisa de Solos (Rio de Janeiro, RJ). $3^{\text {a }}$ Ed. Manual de métodos de análises de Solos. Rio de Janeiro: CNPS. 573 p. 2017.

EMPRESA BRASILEIRA DE PESQUISA AGROPECUÁRIA. Centro Nacional de Pesquisa de Solos (Rio de Janeiro, RJ). $3^{\text {a }}$ Ed. Sistema Brasileiro de Classificação de Solos. Rio de Janeiro: CNPS. 353 p. 2013.

ENSINAS, S. C.; SERRA, A. P.; MARCHETTI, M. E.; SILVA, E. F.; LOURENTE, E. R. P.; PRADO, E. A. F.; MATOS, F. A.; ALTOMAR, P. H.; MARTINEZ, M. A.; POTRICH, D. C.; CONRAD, V. A.; JESUS, M. V.; TÁRIK KADRI, C. E. Cover crops affect the soil chemical properties under no-till system. Australian Journal of Crop Scienc. v. 10, n. 8, p. 1104-1111. 2016.

EUCLIDES, V. B. P.; CARPEJANI, G. C.; MONTAGNER, D. B.; NASCIMENTO JUNIOR, D.; BARBOSA, R. A.; DIFANTE, G. S. Maintaining post-grazing sward height of Panicum maximum (cv. Mombaça) at $50 \mathrm{~cm}$ led to higher animal performance compared with postgrazing height of $30 \mathrm{~cm}$. Grass and Forage Science. v. 73, n. 1, 174-182, 2017.

FOYER, C. H.; LAM, H-M., NGUYEN, H. T.; SIDDIQUE, K. H. M.; VARSHNEY, R. K.; COLMER, T. D.; COWLING, W.; BRAMLEY, H.; MORIS, T. A, HODGSON, J. M. Neglecting legumes has compromised human health and sustainable food production. Nature Plants. v. 2, n.1, p. 1-10, 2016. 
GODINOT, O.; CAROF, M.; VERTES, F.; LETERME, P. SyNE: an improved indicator to assess nitrogen efficiency of farming. Agricultural Systems. v. 127, n. 1, p. 41-52. 2014.

LASSALETTA, L.; BILLEN, G.; GRIZZETTI B.; ANGLADE, J.; GARNIER, J. 50 year trends in nitrogen use efficiency of world cropping systems: the relationship between yield and nitrogen input to cropland. Environmental Research Letters. v. 9, n.1, p. 1-9, 2014.

LIU, E.; YAN, C.; MEI, X.; BING, S. H.; DING, L.; LIU, Q.; LIU, S.; FAN, T. Long-term effect of chemical fertilizer, straw, and manure on soil chemical and biological properties in northwest China. Geoderma. v. 158, n. 1, p. 173-180. 2010.

MAGALHÃES, R. T.; OLIVEIRA, I. P.; KLIEMANN, H. J. Relações da produção de massa seca e as quantidades de nutrientes exportados por Brachiaria brizantha em solos sob o manejo pelo sistema "Barreirão". Pesquisa Agropecuária Tropical. v. 32, n. 1, p. 13-20. 2002.

MALAVOLTA, E. Elementos de nutrição de plantas. Agronômica Ceres, São Paulo. 251 p. 1980.

MOREIRA, L. M.; SANTOS, M. E. R.; FONSECA, D. M.; MARTUSCELLO, J. A.; MORAIS, R. V.; MISTURA, C. Produção animal em pastagem de capim-braquiária adubada com nitrogênio. Arquivo Brasileiro de Medicina Veterinária e Zootecnia. v. 63, n. 4, p. 914-921. 2011.

ŚWITEK, S.; TAKACS, V.; SAWINSKA, Z.; KOSIADA, T.; TRYJANOWSKIA, P. Mineral nitrogen fertilisers remain a crucial factor even in the ecological intensification of agriculture. Acta Agriculturae Scandinavica, Section B - Soil \& Plant Science. v. 69, n. 4, p. 311-316. 2019.

TAIZ, L e ZEIGER E. Fisiologia vegetal. 3rd. edn. Artmed, Porto Alegre. 722p. 2006. 